PROCEEDINGS OF THE

AMERICAN MATHEMATICAL SOCIETY

Volume 129, Number 2, Pages 351-353

S 0002-9939(00)05646-X

Article electronically published on July 27, 2000

\title{
A NOTE ON BRANCHING THEOREMS
}

\author{
KENNETH D. JOHNSON \\ (Communicated by Roe Goodman)
}

\begin{abstract}
Let $G$ be a complex, simply connected semisimple analytic group with $K$ a closed connected reductive subgroup. Suppose $V$ is an irreducible holomorphic $G$-module and $W$ an irreducible holomorphic $K$-module. We prove that $\operatorname{Hom}_{K}(W, V)$ possesses the structure of an irreducible $U(\mathfrak{g})^{K}$ module whenever $\operatorname{Hom}_{K}(W, V)$ is $\neq(0)$. Moreover, $\operatorname{dim}_{K} \operatorname{Hom}_{K}(W, V) \leq 1$ for all $W$ and $V$ if and only if $U(\mathfrak{g})^{K}$ is commutative.
\end{abstract}

\section{INTRODUCTION}

Suppose $G$ is a complex, semisimple, simply connected, analytic group with $K$ a closed, reductive, complex, analytic subgroup. Let $\mathfrak{g}$ and $\mathfrak{k}$ denote the respective Lie algebras of $G$ and $K$ with $U(\mathfrak{g})$ and $U(\mathfrak{k})$ their corresponding enveloping algebras. Set $M(G)$ equal to the set of equivalence classes of irreducible holomorphic $G$ modules, and similarly, let $M(K)$ be the corresponding set of equivalence classes of $K$-modules (as usual we identify elements of $M(G)$ or $M(K)$ with representations of the class). Finally, let $Z=U(\mathfrak{g})^{K}$ be the ring of $A d K$-invariant elements of $U(\mathfrak{g})$.

Given $V \in M(G)$ we have that as a $K$-module

$$
V=\bigoplus_{W \in M(K)} i(W, V) W
$$

where $i(W, V)=\operatorname{dim} \operatorname{Hom}_{K}(W, V)$. We now state the main result of this note.

Theorem. The ring $Z$ is commutative if and only if $i(W, V) \leq 1$ for all $W \in M(K)$ and $V \in M(G)$.

Although this theorem is folklore to anyone who has studied branching results, a proof does not appear in the literature. Our purpose here is to provide a simple proof of this theorem.

Weyl's branching theorems tell us that

$$
\begin{gathered}
i(W, V) \leq 1 \text { if }(G, K)=(S L(n+1, \mathbb{C}), G L(n, \mathbb{C})) \text { or } \\
(G, K)=(\operatorname{Spin}(n+1, \mathbb{C}), \operatorname{Spin}(n, \mathbb{C})) .
\end{gathered}
$$

A result of Knop [K] tells us that $Z$ is commutative only when $(G, K)$ is one of these two cases or a product of them.

Received by the editors September 1, 1998 and, in revised form, April 22, 1999.

1991 Mathematics Subject Classification. Primary 17B35, 22E46; Secondary 22E10.

Key words and phrases. Enveloping algebra, centralizer, module.

(C)2000 American Mathematical Society 


\section{THE PROOF}

Given $V \in M(G)$ and $W \in M(K), \operatorname{Hom}_{K}(W, V)$ possesses the structure of a $Z$ module. If $D \in Z, T \in \operatorname{Hom}_{K}(W, V)$, then $D(T)(w)=D(T w)$ for $w \in W$. Hence $\operatorname{Hom}_{K}(W, V) \otimes W$ becomes a $(Z, K)$-module, and thus

$$
\widetilde{V}=\bigoplus_{W \in M(K)} \operatorname{Hom}_{K}(W, V) \otimes W
$$

has the structure of a $(Z, K)$-module.

Consider the linear evaluation map $E: \widetilde{V} \rightarrow V$ such that $E(T \otimes w)=T(w)$ if $T \in \operatorname{Hom}_{K}(W, V)$ and $w \in W$.

Proposition 2.1. The map $E$ is a $(Z, K)$-isomorphism.

Proof. It is easily seen that $E$ intertwines the action of $Z$, and $K$, and by definition $E$ is an isomorphism.

We now see that $\widetilde{V}$ has the structure of a $G$-module compatible with the given $(Z, K)$-structure. Moreover, $\widetilde{V}$ is equivalent to $V$ as a $G$-module. Let $\pi$ denote the corresponding representation of $G, \mathfrak{g}$ and $U(\mathfrak{g})$ on $\widetilde{V}$.

Proposition 2.2. $\operatorname{End}_{K}(\widetilde{V})=\pi(Z)$.

Proof. Clearly, $\pi(Z) \subset \operatorname{End}_{K}(\widetilde{V})$. Suppose $S \in \operatorname{End}_{K}(\widetilde{V})$. By Burnside's theorem $\pi(U(\mathfrak{g}))=\operatorname{End}(\widetilde{V})$. Hence there is a $D \in U(\mathfrak{g})$ such that $\pi(D)=S$. Since $K$ is reductive, there is a compact analytic group $K_{0} \subset K$ whose Lie algebra is a real form of $\mathfrak{k}$. Then integrating with respect to the normalized Haar integral of $K_{0}$, we have

$$
D_{0}=\int_{K_{0}} \operatorname{Adk}(D) d k
$$

is in $Z$ and $\pi\left(D_{0}\right)=\pi(D)=S$.

Proposition 2.3. $\operatorname{End}_{K}(\widetilde{V})=\operatorname{End}\left(\operatorname{Hom}_{K}(W, V)\right) \otimes I_{W}$.

Proof. Clearly

$$
\operatorname{End}_{K}(\tilde{V})=\bigoplus_{W \in M(K)} \operatorname{End}_{K}\left(\operatorname{Hom}_{K}(W, V) \otimes W\right) .
$$

Since $\operatorname{End}_{K}\left(\operatorname{Hom}_{K}(W, V) \otimes W\right)=\operatorname{End}\left(\operatorname{Hom}_{K}(W, V)\right) \otimes I_{W}$, we are done.

Proof of Theorem 1. If $Z$ is commutative, $\operatorname{End}\left(\operatorname{Hom}_{K}(W, V)\right)$ is commutative for any $V \in M(G)$ and any $W \in M(K)$. Thus $i(W, V) \leq 1$ for any $V \in M(G)$ and any $W \in M(K)$. On the other hand suppose $i(W, V) \leq 1$ for all $W$ and $V$. Since $i(W, V) \leq 1$ for all $V \in M(G)$ and all $W \in M(K), \operatorname{End}\left(\operatorname{Hom}_{K}(W, V)\right)$ is abelian and so $\operatorname{End}_{K}(V)$ is abelian. Thus $[Z, Z]$ is 0 on $V$ for any $V \in M(G)$. From 2.5.7 of [D], $Z$ maps injectively into the direct product of the $\operatorname{End}_{K}(V)(V \in M(G))$. Hence $[Z, Z]=0$, and $Z$ is abelian.

\section{REFERENCES}

[B] Hermann Boerner, Representations of Groups, 2nd edition, North-Holland, Amsterdam, 1970. MR 42:7792

[D] Jacques Dixmier, Enveloping Algebras, North-Holland, Amsterdam, 1977. MR 58:16803b

[J] Nathan Jacobson, Lectures in Abstract Algebra, Vol. II, D. vanNostrand, Princeton, 1953. MR 14:837e 
[K] Friedrich Knop, Der Zentralisator einer Liealgebra in einer einhüllenden Algebra, J. Reine Angew. Math. 406 ((1990)), 5-9. MR 91k:17007]

[W] Hermann Weyl, Theory of Groups and Quantum Mechanics, Dover, New York, 1931.

Department of Mathematics, University of Georgia, Athens, Georgia 30602

E-mail address: ken@alpha.math.uga.edu 